\title{
Bioassays based on molecular nanomechanics
}

\author{
Arun Majumdar \\ Nanoengineering Laboratory, Department of Mechanical Engineering, University of California, Berkeley, CA \\ 94720, USA \\ Tel.: +1 510643 8199; Fax: +1 510642 6163; E-mail: majumdar@me.berkeley.edu
}

\begin{abstract}
Recent experiments have shown that when specific biomolecular interactions are confined to one surface of a microcantilever beam, changes in intermolecular nanomechanical forces provide sufficient differential torque to bend the cantilever beam. This has been used to detect single base pair mismatches during DNA hybridization, as well as prostate specific antigen (PSA) at concentrations and conditions that are clinically relevant for prostate cancer diagnosis. Since cantilever motion originates from free energy change induced by specific biomolecular binding, this technique is now offering a common platform for label-free quantitative analysis of protein-protein binding, DNA hybridization DNA-protein interactions, and in general receptor-ligand interactions. Current work is focused on developing "universal microarrays" of microcantilever beams for high-throughput multiplexed bioassays.
\end{abstract}

Keywords: Molecular nanomechanics, microcantilever beams, bioassay, universal microarrays

\section{Introduction}

It is now fairly well recognized that for diagnosis, monitoring, prognosis, and molecular classification of complex diseases such as cancer, it is necessary to generate molecular profiles and patterns by quantitatively detecting large numbers of biomolecules from serum or tissue samples. To facilitate this, it is necessary to develop high-throughput techniques that can detect a wide variety of molecules. For genetic analysis, DNA microarrays have revolutionized the way genes and gene expressions can be analyzed in a multiplexed manner. Microarray analysis, however, provides only a glimpse of a small but important fraction of the complex molecular machinery involved in a cell. Development of high-throughput techniques that can identify and quantitate proteins and their various modifications and specific interactions has remained a challenge. The most widely used techniques, which include two-dimensional gel electrophoresis and mass spectroscopy, can identify and quantitate individual proteins, but are far from capturing the subtleties of proteins and their complex interactions with various other molecules. What would be ideal are techniques that are sufficiently robust and universal that can detect sub- tle changes in molecular configurations and specific molecular interactions. It is, therefore, important to ask the question: What is common between all specific biomolecular interactions?

It must be recognized that all biomolecular reactions are thermodynamically driven by reduction of free energy of the system. In addition, the reduction of free energy for specific reactions must be much larger than that for non-specific interactions, since otherwise nonspecific reactions would prevail and the complexity of molecular interactions within a cell, which relies of specificity, would be lost. It is, therefore, worth asking the question: Can the free energy change of biomolecular reactions be detected?

It is important to note that thermodynamics is the language common between various physical phenomena that involve exchange of energy and entropy: mechanics, electricity, magnetism, etc. Hence, if the free energy reduction in biomolecular reactions can be translated into another form, it could provide a way for detecting the reaction. Recent experiments have shown that when specific biomolecular reactions occur on one surface of a "diving board" shaped microcantilever beam (see Fig. 1), the cantilever beam bends [1-3]. The cantilever motion is thought to originate from changes in intermolecular nanomechanical 


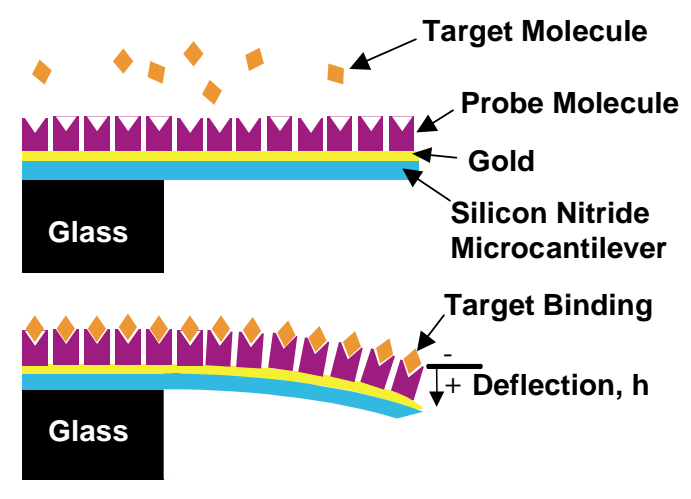

Fig. 1. Specific biomolecular interactions between target and probe molecules alters the intermolecular interactions within a self-assembled monolayer on one side of a cantilever beam. This can produce a sufficiently large torque to bend the cantilever beam and generate motion.

forces, which arise due changes in molecular configurations or charges that are induced by the reactions. From the view point of thermodynamics, the reaction on one surface changes its surface free energy density or surface tension, which produces a differential torque that bends the cantilever. This is an example where the free energy reduction of biomolecular interactions can be translated into increase of mechanical free energy of the cantilever beam. What is critical, however, is the fact that this technique can be used to detect biomolecular reactions under conditions and at levels that are clinically important, examples of which are described in the next section. In addition, because free energy reduction is the driving force for all biomolecular reactions, this could be a universal technique for bioassays. Finally, because microcantilever beams are readily amenable to microarray formation, one could envision multiplexed bioassays for high-throughput analysis.

The goal of this paper is to review the past research in this field and provide a look at the near future. Section 2 describes past single-cantilever experiments on DNA hybridization and single base pair mismatch detection, as well as on quantitative detection of prostate specific antigen (PSA). Section 3 describes our effort on microarray development. Section 4 concludes with some noteworthy points that emphasize the technological relevance of this technique.

\section{Single cantilever experiments}

In this section, past experiments [1-7] using single cantilever analysis are described. The set-up for these experiments is quite common, and hence description of only one type of set up, the one used in our laboratory, is provided.

\subsection{Experimental set-up}

Figure 2 shows the experimental setup. It consisted of a transparent fluid cell within which a gold-coated silicon nitride ( $\mathrm{Au} / \mathrm{SiNx}$ ) cantilever was mounted. The cantilevers used were 200-600 $\mu \mathrm{m}$ long, $0.5 \mu \mathrm{m}$ thick, and $20-40 \mu \mathrm{m}$ wide. The fluid cell formed a liquid reservoir about $100 \mu \mathrm{l}$ in volume that was connected to an inlet and an outlet fluid port. To detect cantilever deflections, a low-power $(\approx 1 \mathrm{~mW})$ laser beam was reflected off the cantilever and was focused onto a position-sensitive diode (PSD). Such a set up is commonly used in atomic force microscopes. To eliminate thermomechanical motion of the $\mathrm{Au}-\mathrm{SiNx}$ bimaterial cantilever due to temperature fluctuations, the glass slide and the fluid cell were mounted on thermoelectric coolers such that the temperature of the fluid cell could be controlled to $25 \pm 0.05^{\circ} \mathrm{C}$. The experiment started by first placing a $\mathrm{Au} / \mathrm{SiNx}$ cantilever in a fluid cell and then injecting a solution of sodium phosphate buffer $(\mathrm{PB})$ at $\mathrm{pH} \sim 7.0$ (always with the same $\mathrm{pH}$ but maybe different ion concentration for different experiments) into the cell. The cantilever was equilibrated in the PB buffer until a stable base line was obtained. The next step was to immobilize the probe molecules, which were resuspended in the same PB buffer used to equilibrate the cantilever, on the cantilever surface. After the immobilization was completed (typically about two hours at room temperature), the fluid cell was washed thoroughly with the PB buffer to be used for hybridization. Then the cantilever was equilibrated in the same PB buffer (as that to be used for hybridization) again until a stable baseline was obtained. Finally, injection of a solution of target molecules (resuspended in the same PB buffer) followed. The cantilever motion was optically monitored at both the immobilization and probe-target binding steps. For each experiment, a new cantilever was used. The error induced by variations in the geometry of the cantilever (length, width and thickness) and the position of the focused laser spot at the end of the cantilever was found to be within $\pm 5-10 \%$.

Fritz et al. [3] monitored the motion of two cantilevers simultaneously, one functionalized with probe biomolecules and other without any biomolecules. They then obtained the difference signal in order to eliminate thermomechanical motion and cantilever drift, and capture only the motion induced by biomolecular reactions. This is a better approach for measurements as long as the drift and the thermomechanical response of the two cantilevers are the same. 


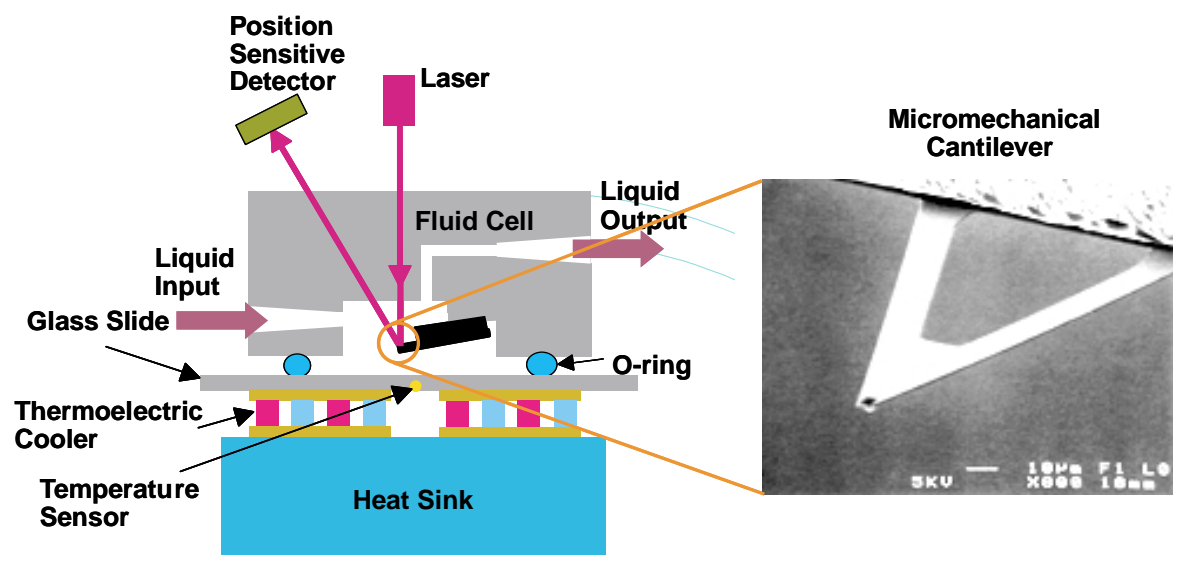

Fig. 2. Schematic diagram of the experimental setup showing a fluid cell within which a microcantilever beam was mounted. The scanning electron micrograph on the right shows the geometry of a Au-coated silicon nitride cantilever beam that was $200 \mu \mathrm{m}$ long, $0.5 \mu \mathrm{m}$ thick, and with each leg $40 \mu \mathrm{m}$ wide. To measure the cantilever deflection, a laser was reflected off the back of the cantilever and focused onto a position-sensitive detector. The reagents were injected into the fluid cell using the liquid ports. The fluid cell was mounted on a temperature controlled glass slide.

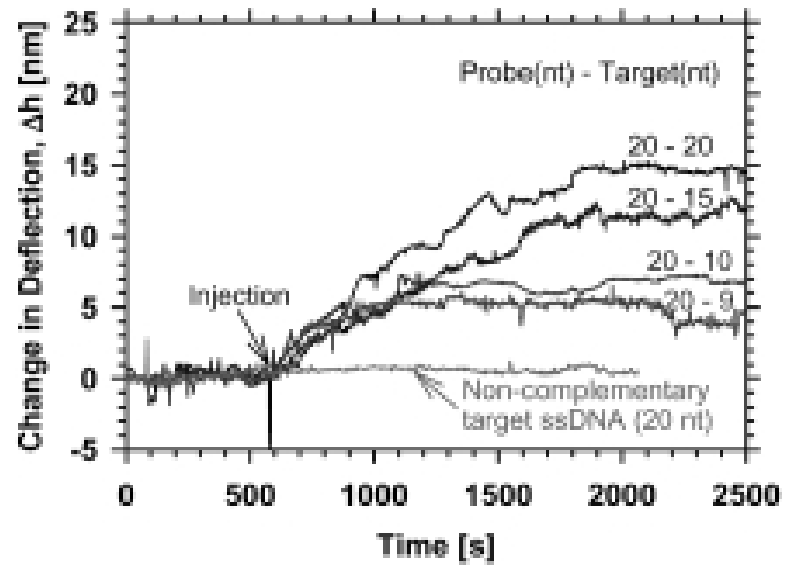

Fig. 3. Changes in Au-Si cantilever deflection due to hybridization of a probe ssDNA ( $50 \mathrm{ng} / \mu 1$ or $8 \mu \mathrm{M}$ concentration) in the distal end with complementary target ssDNA of different lengths - $20 \mathrm{nt}, 15 \mathrm{nt}$, $10 \mathrm{nt}$, and $9 \mathrm{nt}(40 \mathrm{ng} / \mu \mathrm{l}$ or $3-6 \mu \mathrm{M}$ concentration). Also shown is the absence of cantilever deflection for a non-complementary target ssDNA. The data clearly suggests that differences in nanomechanical motion due to one nucleotide difference in length can be observed.

\subsection{DNA hybridization}

Single stranded DNA (ssDNA) can be immobilized using gold-thiol strong binding on one side of a cantilever by coating that side with gold and using a thiol linker at one end of ssDNA. ssDNA bound to the cantilever acts as the probe (or receptor) molecule for the target complementary strands. After immobilizing the probe ssDNA, a solution containing complementary target ssDNA was injected into the fluid cell at the same PB concentration that was used to immobilize the probe ssDNA. Figure 3 shows the deflection profiles for the hybridization reactions where the probe ssDNA was $20 \mathrm{nt}$ long and the complementary target ssDNA were of four different lengths (20 nt, $15 \mathrm{nt}, 10 \mathrm{nt}$ and $9 \mathrm{nt}$ ) and chosen to be distally complementary [4]. The nanomechanical signal was sufficiently sensitive to detect single nucleotide length differences. We have also performed hybridization experiments using 30-50 nt long DNA and the results have shown very similar trends. The observation that the cantilever bent upwards in all cases suggests that hybridization relieved the compressive stress created during immoblization of thiolated probe ssDNA. To confirm that the signals were due to hybridization, a solution of a non-complementary target ssDNA was used and was found to produce no deflection.

It was found that the amount of deflection during ssDNA immobilization and DNA hybridization depended on the ion concentration in the solution. This suggests that electrostatic repulsive forces between neighboring DNA molecules must play a role in cantilever motion. Because each nucleotide carries a net negative charge due to the presence of a phosphate group, one would expect the hybridization to cause even more repulsion due to the presence of additional negative charge. However, the data in Fig. 3 clearly indicates that hybridization always relieved the stress and produced upward cantilever motion. Therefore, electrostatic or steric repulsion alone cannot explain the behavior.

It is well known [8] that end-grafted polymers, at sufficiently high grafting densities, adopt stretched conformations in order to reduce intersegment interactions resulting from steric or electrostatic repulsion. This chain stretching is, however, entropically unfavorable. 


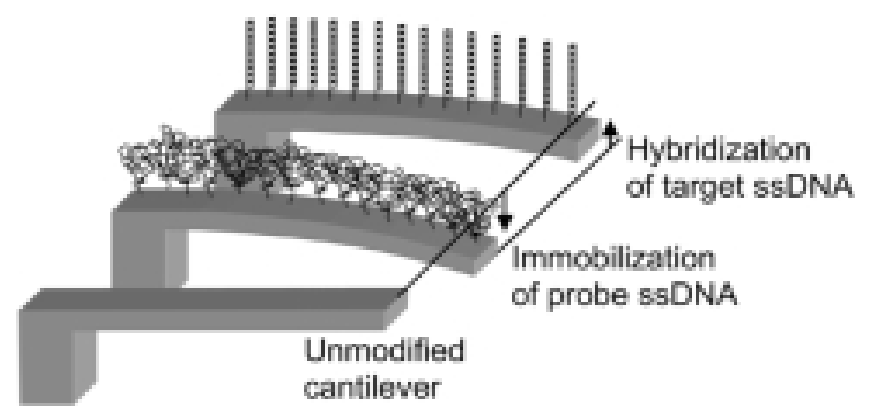

Fig. 4. Schematic diagram illustrating the mechanism of motion generation due to DNA immobilization and hybridization. Immobilization of ssDNA on the top surface bends the cantilever down. The persistence length of ssDNA is $7.5 \mu$, and this flexibility provides an entropic driving force for forming curved interfaces. Hybridization increases the persistence length to about $50 \mathrm{~nm}$, which significantly reduces the conformational entropic driving force, thereby reducing the importance of curvature producing an upward cantilever motion.

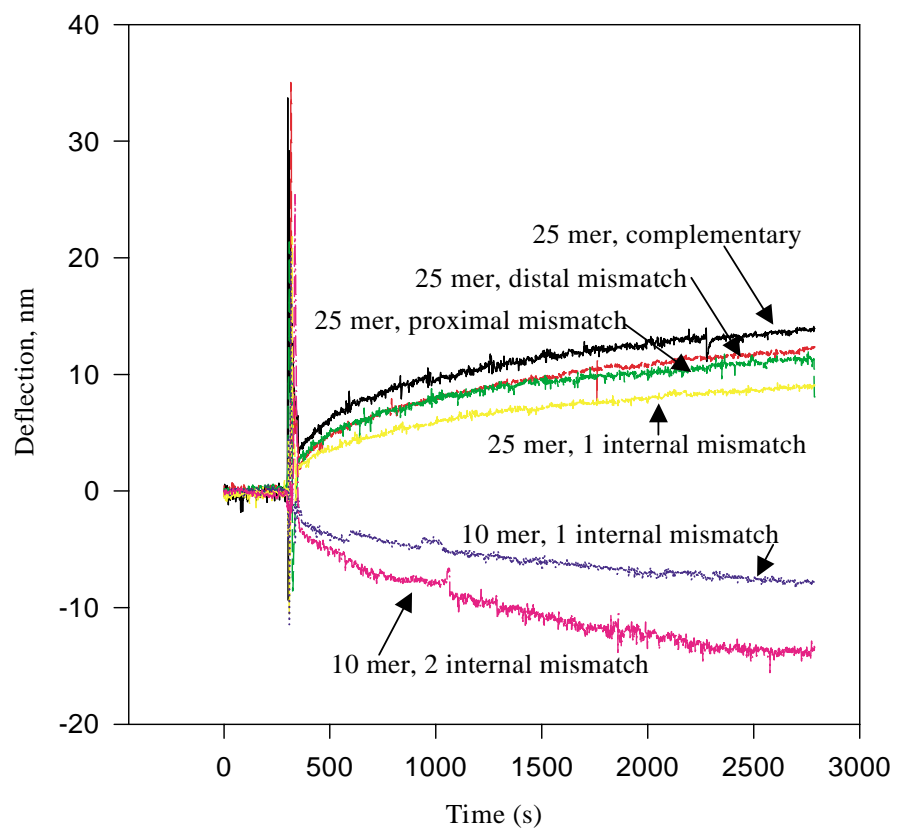

Fig. 5. Cantilever deflection for 25 mer thiolated probe ssDNA hybridized with complementary and mismatch target oligonulceotides.

As is well known [9], the entropic penalty can be alleviated by adsorption onto a convex surface. This is because the curvature allows each chain to adopt more conformations (hence acquiring greater conformational entropy) as the distance from the surface increases. At the ionic strengths of our experiments $(0.05-1 \mathrm{M})$, the persistence length of ssDNA is $0.75 \mathrm{~nm}$ [10], which corresponds to approximately two nucleotides. Thus, for sufficiently long ssDNA molecules, the conformational entropy gain by forming a curved interface is significant. Thus, in addition to electrostatic intersegment repulsion, there is an entropic driving force for bending the cantilever downwards. These forces are balanced by the strain energy of bending the cantilever, leading to an equilibrium value of curvature and cantilever deflection. The persistence length of double-stranded DNA (dsDNA) formed after hybridization is 50-80 nm [11]. Thus, upon hybridization, the dramatically increased chain stiffness makes the entropic driving force to form a curved interface unimportant, as illustrated in Fig. 4. Therefore, the cantilever strain energy and intersegment repulsion are balanced at a smaller curvature (i.e. smaller deflection).

Although this seemed like a plausible explanation earlier [4], further in-depth studies have shown that this may not be the complete picture [12]. It has been found that osmotic effects (entropic force for the ions surrounding DNA) are equally important. In fact, be- 


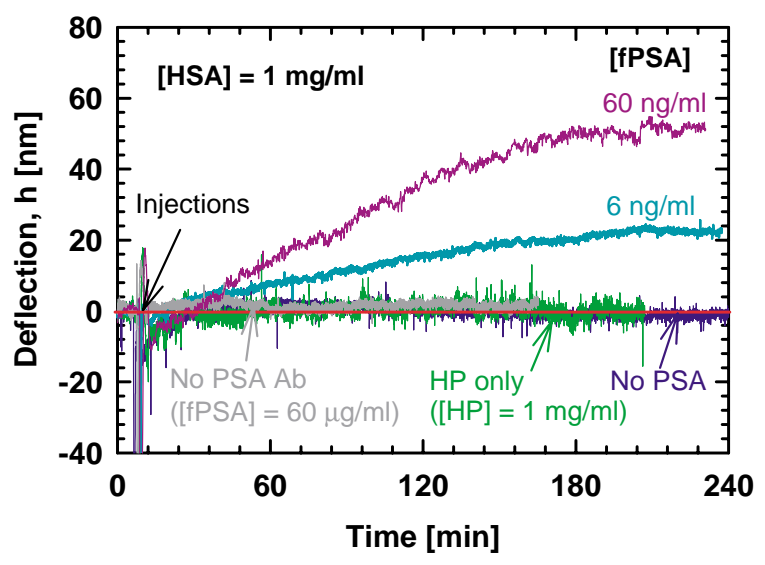

Fig. 6. Specificity of free PSA (fPSA) detection against a high background of human serum proteins, namely, human serum albumin (HSA) and human plasminogen (HP), both at concentrations of $1 \mathrm{mg} / \mathrm{ml}$. The cantilevers used were $200 \mu \mathrm{m}$ long and $0.5 \mu \mathrm{m}$ thick and made of silicon nitride.

cause the distance between neighboring DNA strands in a self-assembled monolayer is on the order of 3$5 \mathrm{~nm}$, their hydration shells can overlap. Hence, hydration forces can become extremely important, and perhaps dominant in generating the nanomechanical forces. Finally, it was concluded that because hydration, osmotic and conformational entropic forces are highly non-linear with intermolecular distance, the disorder in the self-assembly of probe molecules is an important parameter that controls the level of cantilever deflection. It is clear that although we understand some aspects of the origins of cantilever motion, much remains to be learnt.

Figure 5 shows cantilever deflection versus time for DNA hybridization [5], in which the probe ssDNA was $25 \mathrm{nt}$ long. Target ssDNA of $25 \mathrm{nt}$ long was used, but in some cases a single base pair mismatch was introduced either in the proximal or distal ends, or in the middle. It can be seen that single base pair mismatches can be mechanically distinguished. What is intriguing, however, is the fact that when the target ssDNA length is reduced to $10 \mathrm{nt}$, then introducing a single base pair mismatch moved the cantilever downwards (opposite to the other cases), while introducing two base pair mismatches produced almost the double the downwards deflection. The origins of this are not yet understood.

The fact that single base pair mismatches can be mechanically detected opens the possibility of analyzing single nucleotide polymorphisms (SNPs) that important not only in detecting diseases for a wide variety of genomic studies. It is also important to point out that while DNA microarrays that rely on fluorescent detection require probe DNAs that are typically 20 mer in length, the cantilever based technique might allow detection of shorter oligonucleotides, which could be important in many genomic applications.

\subsection{Antigen-antibody reactions}

Antigen-antibody binding reactions are a class of highly specific protein-protein interactions. When antibody molecules were immobilized to one surface of a cantilever, it has been shown that specific binding between antigens produced cantilever deflection [13]. In the last two years, joint work between three laboratories ${ }^{1}$ have shown the prostate specific antigen (PSA) can be detected at concentrations and under conditions that are clinically relevant [6]. It should be noted that PSA that is detectable in serum has proven to be an extremely useful marker for early detection of prostate cancer and in monitoring patients for disease progression and treatment efficacy.

To detect PSA using cantilevers, a single polyclonal anti-PSA antibody was covalently linked to cantilever surface. The cantilevers used were made of silicon nitride $(\mathrm{SiNx})$ with a thin coating of gold $(\mathrm{Au})$ on one side and with length of $200 \mu \mathrm{m}$, thickness of $0.5 \mu \mathrm{m}$, with each leg width of $20 \mu \mathrm{m}$. A Au film was used to immobilize the PSA antibody to the cantilever through thiol chemistry. Figure 6 shows cantilever deflection as a function of time for different concentration of PSA in a mixture of human serum albumin (HSA) and human plasminogen (HP) as simulated background, each at a high concentration of $1 \mathrm{mg} / \mathrm{ml}$. Similar results were observed against a background of bovine serum albumin (BSA) at $1 \mathrm{mg} / \mathrm{ml}$. The specificity of PSA detection was verified by exposing PSA to a cantilever without any PSA antibody, where negligible deflection was found. When a cantilever functionalized with PSA antibody was exposed to HSA and HP in the absence of fPSA, no significant cantilever deflection was observed. This indicated the high specificity between PSA antigen-antibody binding in the background of HSA and HP.

As the data indicates, it took considerable time for the deflection to reach steady state. This was largely attributed to the time required for the PSA molecules to diffuse in a fluid cell. The time could be drastically re-

\footnotetext{
${ }^{1}$ Arun Majumdar, Mechanical Engineering Dept., UC Berkeley; Thomas Thundat, Life Sciences Division, Oak Ridge National Lab; Ram Datar and Richard Cote, Dept. of Pathology, U. Southern California.
} 


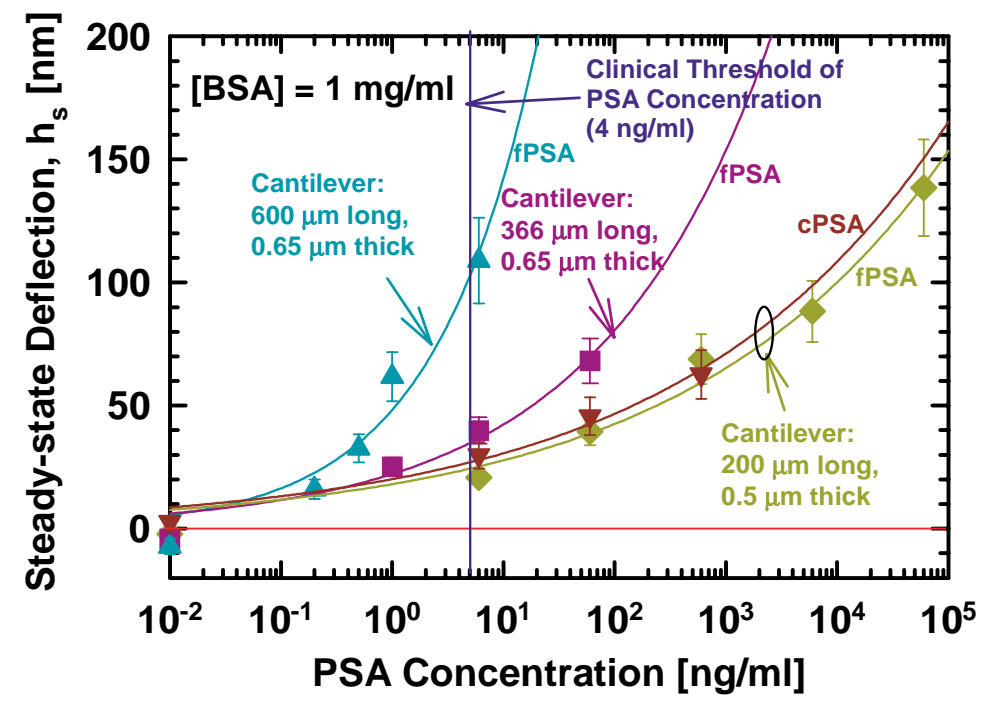

Fig. 7. Steady state cantilever deflections as a function of PSA concentrations for three different cantilever geometries. Note that longer cantilevers produce larger deflections for the same PSA concentration, thereby providing higher sensitivity. Using $600 \mu \mathrm{m}$ long and $0.65 \mu \mathrm{m}$ thick silicon nitride cantilevers, it was feasible to detect fPSA concentration of $0.2 \mathrm{ng} / \mathrm{ml}$.
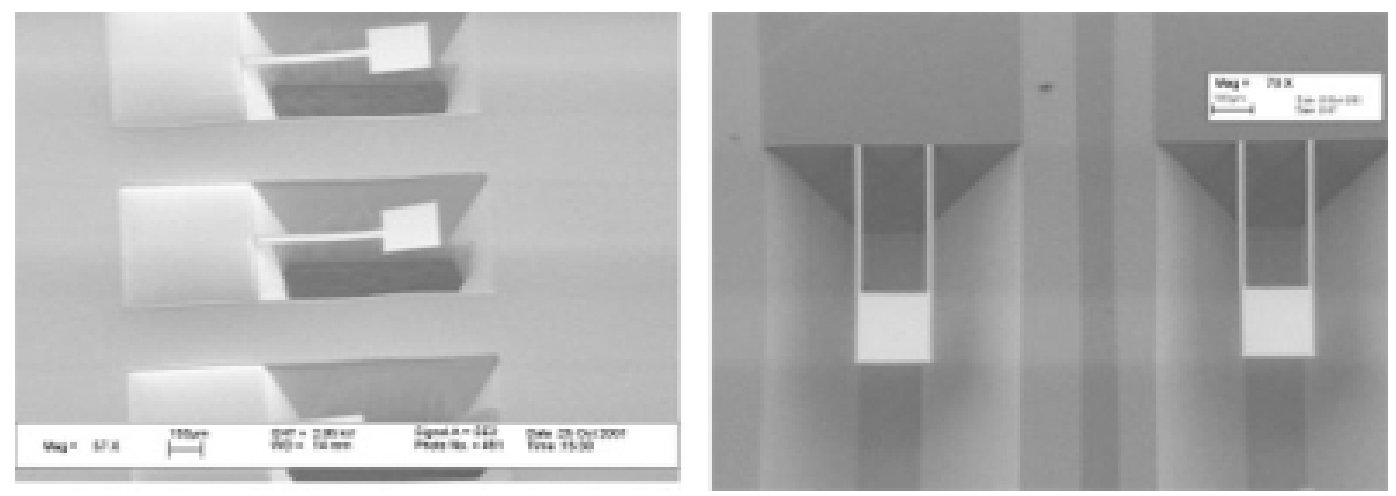

Fig. 8. Electron micrographs of two different chips containing arrays of microcantilever beams. In one, the cantilevers have only one arm, while in the other the cantilevers have two arms. In both cases, a square paddle at the end of the cantilever was used for reflecting light.

duced if the fluid cell is reduced in size (see Section 3). What is important, however, is the observation that the steady-state deflection depended on the concentration of PSA in solution. Figure 7 plots the steady-state cantilever deflection as a function of PSA concentration for three different cantilever lengths. It can be observed that longer cantilevers are more sensitive and produce larger deflections for the same PSA concentrations. What is critical is the demonstration that PSA can be detected at the lowest level of $0.2 \mathrm{ng} / \mathrm{ml}$, which is comparable to other techniques such as ELISA. It is also noteworthy that the clinical threshold for detection of prostate cancer is $4 \mathrm{ng} / \mathrm{ml}$. Wu et al. [6] have provide detailed discussion on the advantages of the cantilever based technique compared to ELISA.

\section{Cantilever microarrays}

The experiments in Section 2 clearly demonstrate that nanomechanical detection of biomolecules can be a useful approach for biomolecular analysis. However, all the results so far have been derived from single cantilever experiments. The true merit of this technology will be realized if one could develop microcantilever arrays for multiplexed high-throughput bioassays. Our group and those of a few others have started developing microcantilever arrays for this purpose. In this section, a brief synopsis of our current activity is provided, while a detailed discussion will appear in a separate publication [13]. 

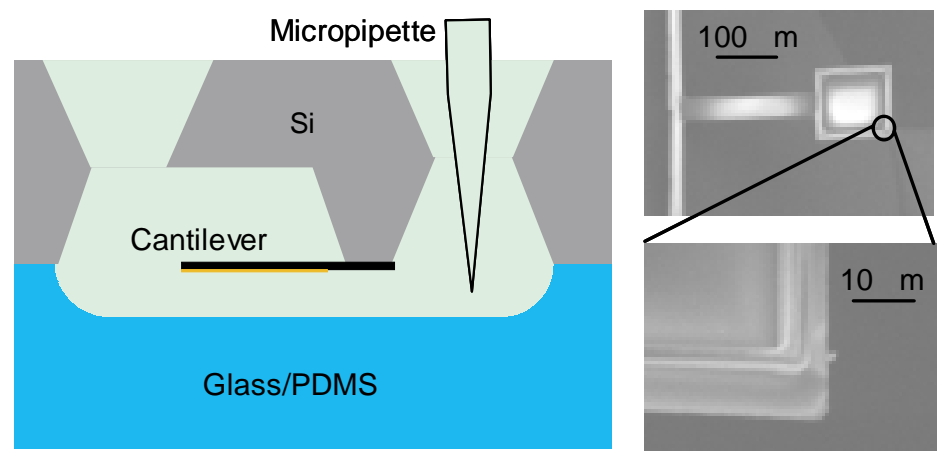

Fig. 9. A schematic of the cantilever and the reaction chamber. A micro reaction chamber is integrated on the chip for each cantilever to allow separate functionalization. Each chamber has one inlet and one outlet to promote bubble-free filling. (b) SEM pictures of a cantilever and its paddle. The cantilever has a $200 \mu \mathrm{m} \times 40 \mu \mathrm{m}$ leg and a $100 \mu \mathrm{m} \times 100 \mu \mathrm{m}$ paddle for optical readout. The ridge on the paddle provides rigidity to the paddle.
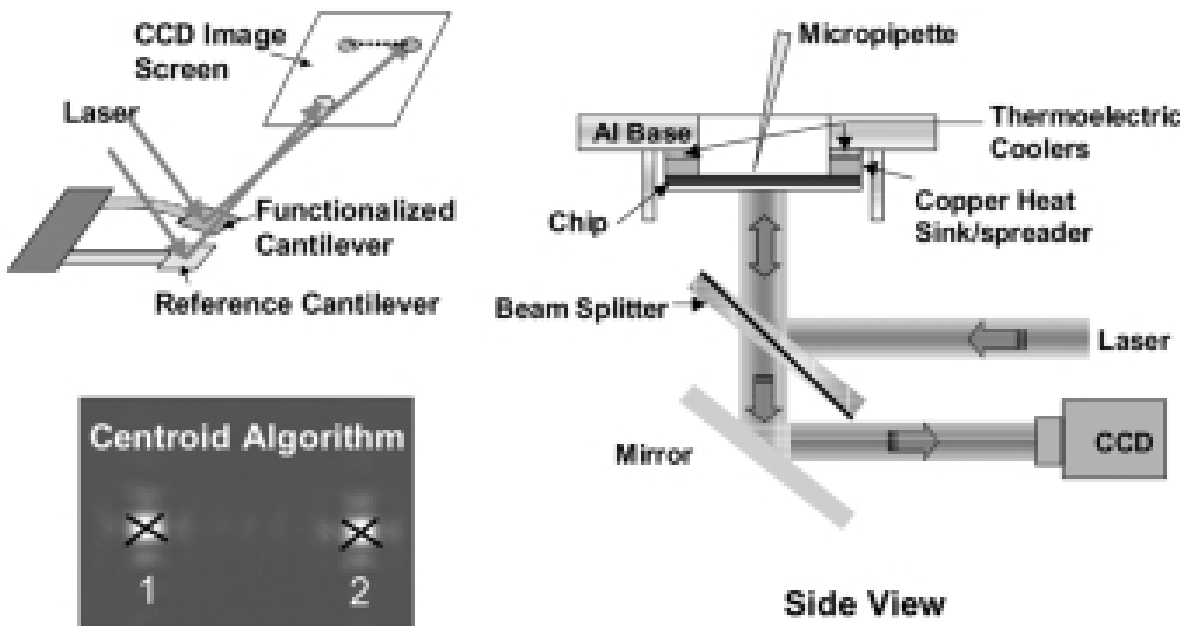

Side View

Fig. 10. A schematic of the optical readout system. An expanded laser beam illuminates the microarray. The paddle at the end of a cantilever works as a mirror to reflect the laser beam. When a cantilever deflects, the paddle changes its angle so the reflected spot shifts on the CCD screen. Two reflection spots are shown.

Figure 8 shows electron micrographs of two different chips, each containing an array of microcantilevers. Using traditional bulk and surface micromachining techniques, the array was fabricated on a silicon substrate with a glass or a PDMS cover. The microarray integrates a reaction chamber with each cantilever to allow for individual cantilever functionalization specific to the target analyte. The reaction chambers have one inlet and one outlet to promote chamber filling. The chambers are made sufficiently small $(1 \mu \mathrm{l})$ such that diffusion times of large biopolymers will be on the order of a few minutes. Currently, a micropipette is used to inject fluid into the reaction chamber (Fig. 9). At the free end of the cantilever, a rigid paddle acts as a mirror surface for the optical readout method described later. We have preferred to use PDMS cover instead of a glass one, since the latter requires a high-temperature bonding process that can warp the cantilever while PDMS bonding is a room temperature process. The PDMS cover is made of Sylgard ${ }^{\circledR} 184$ Silicone Elastomer. It is fabricated using a silicon wafer mold. The cover is then aligned with the silicon chip to form individual reaction chambers. The microcantilever array is finally subjected to oxygen plasma to make the PDMS surface hydrophilic and then immersed and stored in clean DI water.

Simultaneous detection of deflections of hundreds or thousands of cantilevers with 1-5 nm resolution requires thoughtful design. So far we have opted for an optical technique. If there are $\mathrm{N}$ cantilevers, one can use $\mathrm{N}$ sources and 1 detector, 1 source and $\mathrm{N}$ detectors, or $\mathrm{N}$ sources and $\mathrm{N}$ detectors. We have selec- 
tion the second option, where we use one laser whose beam is expanded to be incident on the whole microarray. Reflection of the laser from the reflector paddle of each cantilever is then directed towards a CCD camera, which monitors the laser spots reflected from multiple cantilevers. Figure 10 shows a schematic diagram of the optical system. Currently, we are performing multiplexed bioassays using this array system, the results of which will be published elsewhere.

\section{Conclusions}

Recent experiments have shown that when specific biomolecular reactions are confined to one surface of a diving-board shaped microcantilever beam, the changes in intermolecular nanomechanical forces generate sufficient torque to bend the cantilever beam. In this paper, past research on detection of DNA hybridization, single base pair mismatch, as well as quantitation of prostate specific antigen (PSA) is reviewed. The origins of cantilever is discussed and it is suggested that since cantilever motion is generated due free energy reduction on the cantilever surface, and because free energy reduction is common to all reactions, this technique can offer a common platform for label-free detection of various types of receptor-ligand biomolecular binding. It is argued, however, that the true potential of this approach can only be realized through the development of microcantilever arrays for multiplexed high-throughput bioassays. Our initial progress in this area is also briefly discussed.

\section{Acknowledgements}

This work was supported through grants from the National Cancer Institute, Department of Energy and DARPA. The author is greatly indebted to his collaborators: Thomas Thundat, Karolyn Hansen, and Haifeng Ji from Oak Ridge National Lab; Ram Datar and Richard Cote from University of Southern California; Michael Hagan and Arup Chakraborty from University of California, Berkeley. This work could not have been possi- ble without the hard work, innovation, and persistence by the author's graduate students: Guanghua Wu, Min Yue, Srinath Satyanarayana, Daniel Dedrick and Henry Lin. The author will always remain grateful for their tremendous contributions.

\section{References}

[1] T. Thundat, P.I. Oden and R.J. Warmack, Microcantilever sensors, Microscale Thermophys. Eng. 1 (1997), 185-199.

[2] R. Raiteri, G. Nelles, H.-J. Butt, W. Knoll and P. Skladal, Sensing biological substances based on the bending of microfabricated cantilevers, Sens. Actuators B 61 (1999), 213-217.

[3] J. Fritz, M.K. Baller, H.P. Lang, H. Rothuizen, P. Vettiger, E. Meyer, H.J. Guntherodt, Ch. Gerber and J.K. Gimzewski, Translating biomolecular recognition into nanomechanics, Science 288 (2000), 316-318.

[4] G. Wu, H. Ji, K. Hansen, T. Thundat, R. Datar, R. Cote, M.F. Hagan, A.K. Chakraborty and A. Majumdar, Origin of nanomechanical cantilever motion generated from biomolecular interactions, Proceedings of National Academy of Science 98 (2001), 1560-1564.

[5] K. Hansen, H. Ji, G. Wu, R. Datar, R. Cote, A. Majumdar and T. Thundat, Cantilever-based optical deflection assay for discrimination of DNA single-nucleotide mismatches, Analytical Chemistry 73 (2001), 1567-1571.

[6] G. Wu, R. Datar, K. Hansen, T. Thundat, R. Cote and A. Majumdar, Bioassay of prostate specific antigen (PSA) using microcantilevers, Nature Biotechnology 19 (2001), 856-860.

[7] G. Wu, Nanomechanical biosensor for high throughput genomic and proteomic analysis, Ph.D. Dissertation, University of California, Berkeley, 2001.

[8] S.S. Patel and M. Tirrell, Measurement of forces between surfaces in polymer fluids, Ann. Rev. Phys. Chem. 40 (1989), 587-635.

[9] C.M. Wijmans and E.B. Zhulina, Polymer brushes at curved surfaces, Macromolecules 26 (1993), 7214-7224.

[10] S.B. Smith, Y. Cui and C. Bustamante, Overstretching BDNA: The elastic response of individual double-stranded and single-stranded DNA molecules, Science 271 (1996), 795799.

[11] C.G. Baumann, S.B. Smith, V.A. Bloomfield and C. Bustamante, Ionic effects on the elasticity of single DNA molecules, Proc. Natl. Acad. Sci. 94 (1997), 6185-6190.

[12] M.F. Hagan, A. Majumdar and A.K. Chakraborty, Nanomechanical forces generated by surface grafted DNA, J. Phy. Chem. (in press).

[13] M. Yue, D.E. Dedrick, S. Satyanarayana and A. Majumdar, Microcantilever arrays for multiplexed biomolecular analysis, Proc. ASME IMECE, New Orleans, 2002, in press. 


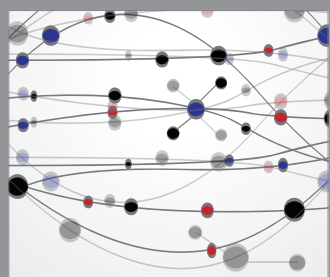

The Scientific World Journal
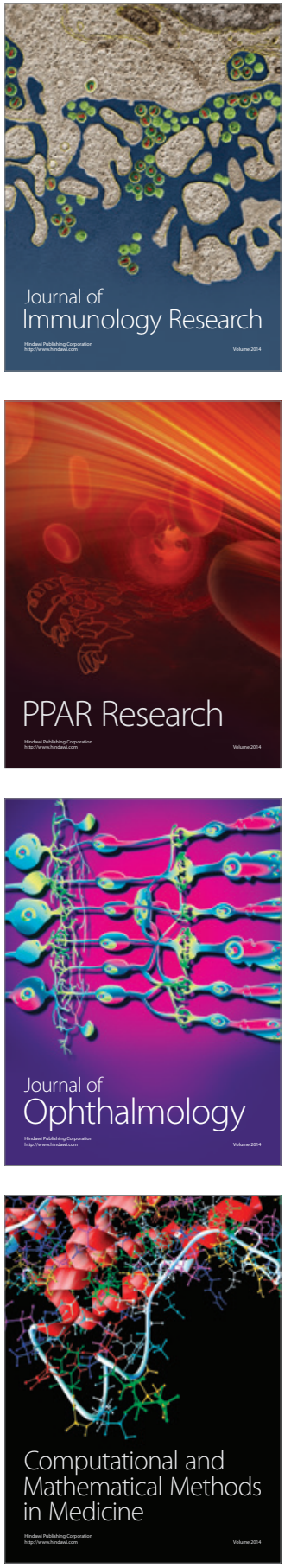

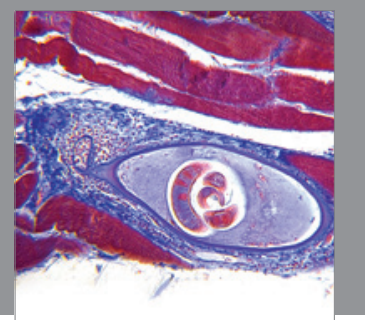

Gastroenterology

Research and Practice
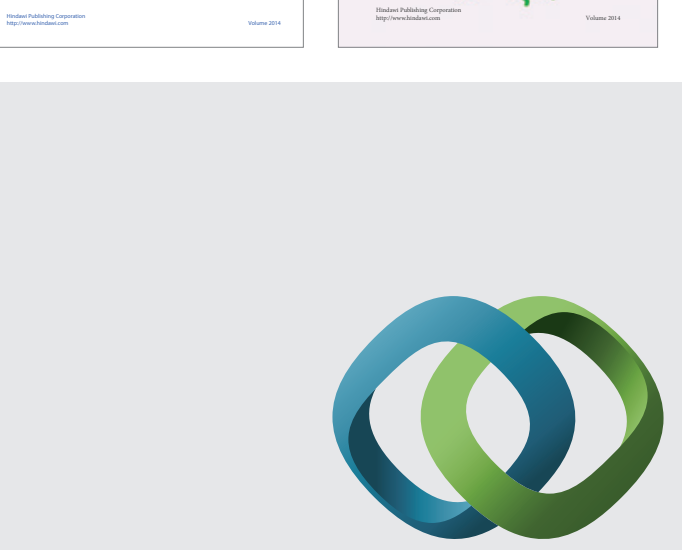

\section{Hindawi}

Submit your manuscripts at

http://www.hindawi.com
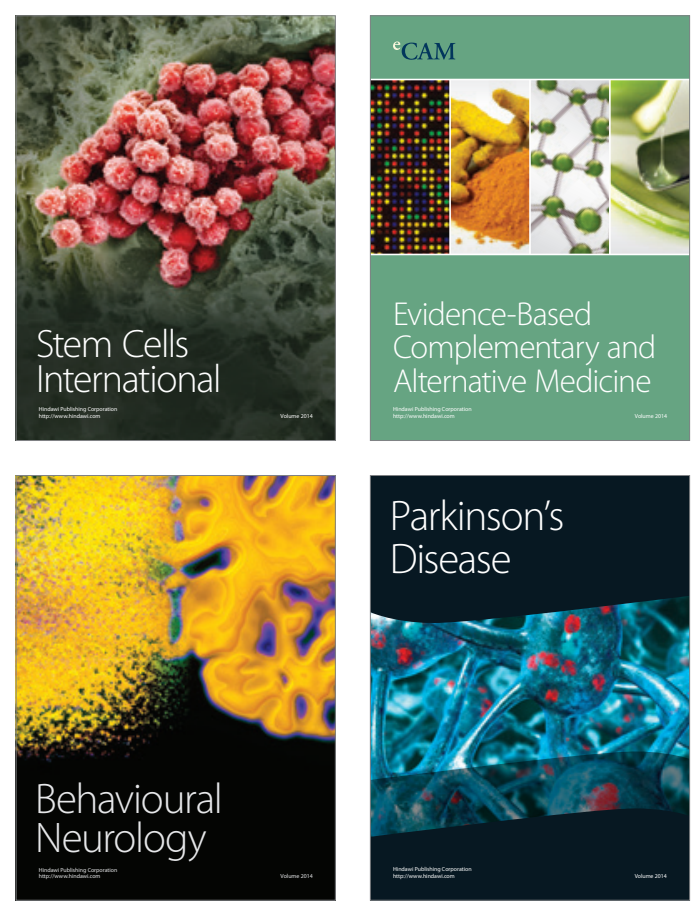

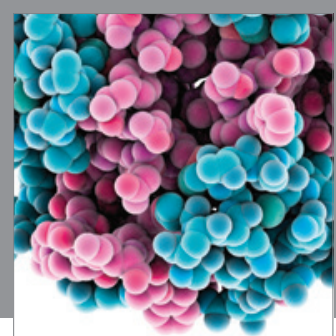

Journal of
Diabetes Research

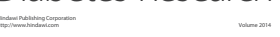

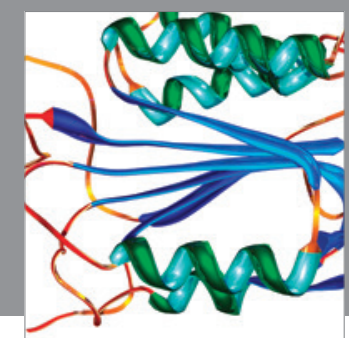

Disease Markers
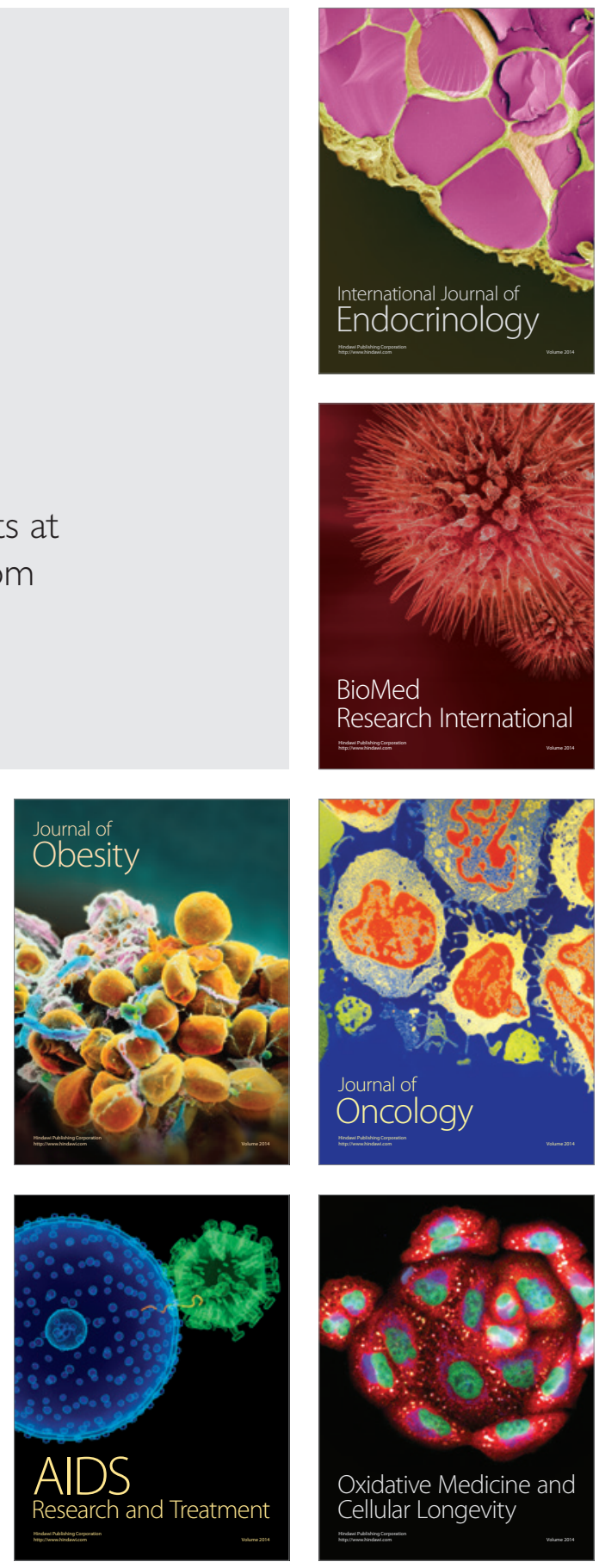